\title{
BLOW-UP SCENARIOS FOR 3D NSE EXHIBITING SUB-CRITICALITY WITH RESPECT TO THE SCALING OF ONE-DIMENSIONAL LOCAL SPARSENESS.
}

\author{
Z. BRADSHAW AND Z. GRUJIĆ
}

\begin{abstract}
It is shown that, if the vorticity magnitude associated with a (presumed singular) three-dimensional incompressible Navier-Stokes flow blows-up in a manner exhibiting certain time dependent local structure, then time independent estimates on the $L^{1}$ norm of $|\omega| \log \sqrt{1+|\omega|^{2}}$ follow. The implication is that the volume of the region of high vorticity decays at a rate of greater order than a rate connected to the critical scaling of one-dimensional local sparseness and, consequently, the solution becomes sub-critical.
\end{abstract}

\section{INTRODUCTION}

The purpose of this article is to present blow-up scenarios under which Leray solutions to the 3D NavierStokes equations (3D NSE) behave sub-critically with respect to the critical scaling of one-dimensional local sparseness. In order to contextualize our discussion we begin with several general remarks. Speaking informally, there is a scaling-gap between known a priori finite quantities on one hand, e.g. $\sup _{0<t<T}\|u\|_{2}^{2}$ and $\sup _{0<t<T}\|\nabla u\|_{1}$, and, on the other hand, quantities with respect to which regularity can be conditioned, e.g. $\sup _{0<t<T}\|u\|_{3}^{3}$ and $\sup _{0<t<T}\|\nabla u\|_{2}^{2}$. Under the natural scaling for 3D NSE,

$$
u(x, t) \mapsto \frac{1}{\lambda} u_{\lambda}\left(\frac{x}{\lambda}, \frac{t}{\lambda^{2}}\right),
$$

we see that the a priori controlled quantities exhibit sub-critical scaling - our examples scale as $\lambda^{1}$ - while those sufficient for regularity scale critically as $\lambda^{0}$. This mismatch is referred to as the "scaling-gap" and indicates the Navier-Stokes problem is super-critical.

A theme apparent in many regularity results is the inclusion of premises that explicitly bridge the scaling gap. For example, a significant result in regularity theory was non-existence of backward-in-time self-similar blow-up (cf. [15, 19, 23]). Because self-similar solutions are scaling invariant in virtue of their construction, their study is effectively a restriction to a class of critical solutions. Indeed, self-similar solutions satisfy a scaling invariant point-wise bound, ess $\sup \left(\left(\left|x-x_{0}\right|+\sqrt{T-t}\right)|u(x, t)|\right)<\infty$, where the essential supremum is taken over an appropriate parabolic cylinder. Regularity of solutions satisfying this estimate has been affirmed if the solution is additionally assumed to be axisymmetric (cf. [3, 4, 17, 20]) but the general case remains an open problem. Regarding the axisymmetric case, the theme originally referenced is again apparent: first, the flow is assumed to exhibit some feature which is critical and, second, additional restrictions are identified from which regularity follows. Similar examples, in particular those from the $\epsilon$-regularity theory, require additional conditions on the smallness of the presumed finite scale-invariant quantity.

In two recent complementary publications, [8, 13], a new dynamic approach to bridging the scaling gap is elucidated. The physical motivation is the persistence (in the average sense) of the axial lengths of vortex filaments. This picture is supported in experimental and numerical studies and by a mathematical result which is the main result of [8]. By considering this length persistence and the decay rate for the volume of the region of intense vorticity, a connection is found between the scaling of the latter quantity and the critical scaling of one-dimensional local sparseness. As this argument provides the context for our own results we make the matter more precise.

The rigorous regularity criteria is presented in [13]. In that paper, regularity of mild solutions to 3D NSE with $L^{\infty}\left(\mathbb{R}^{3}\right)$ initial data is conditioned on a geometric measure-type criteria involving one-dimensional linear sparseness of the super-level sets of the vorticity. The implication is that the critical scaling for the local 
one-dimensional sparseness of the region of intense vorticity is of order $c_{0}\|\omega(t)\|_{\infty}^{-1 / 2}$. The proof of this geometric measure-type regularity criteria is based on an interplay among the diffusion, the basic symmetries present in the 3D NSE and geometric properties of the harmonic measure (resulting in strong anisotropic diffusion); this in turn draws on the ideas exposed in [12]. On the other hand, the a priori estimates on the $L^{1}$ norm of the vorticity found in [6, 18] imply the volume of the region where vorticity magnitudes are high decays according to,

$$
\operatorname{Vol}\left(\Lambda_{t}\left(\frac{1}{c_{1}}\|\omega(t)\|_{\infty}\right)\right) \leq \frac{c_{2}}{\|\omega(t)\|_{\infty}}
$$

where $\Lambda_{t}(y)=\{x:|\omega(x, t)| \geq y\}$. Interestingly, if the region of intense vorticity corresponds precisely to the space occupied by filamentary vortex structures and the length of these filaments is non-decreasing that is, pinned to the characteristic length scale of a turbulent region - then the anti-axial diameters of these filaments exhibit a rate of decay of order at least $c_{3}\|\omega(t)\|_{\infty}^{-1 / 2}$, which matches the critical scaling for local one-dimensional sparseness. It is in this sense that the problem is rendered critical. That the vortex filaments have persistent lengths (in the average) which are comparable to the scale of the turbulent region is not yet rigorously established but is supported by numerical evidence as well as a mathematical evidence which is the prime consideration of [8].

Even assuming the soundness of the above argument it is not immediately evident that regularity follows (it would if one could reconcile the possible difference between $c_{0}$ and $c_{3}$ ). The present paper partially overcomes this by illustrating two blow-up scenarios in which the region of intense vorticity decays at a faster rate than the critical rate discussed in [13, 8], thereby rendering the constants irrelevant. In particular, we are interested in concluding,

$$
\operatorname{Vol}\left(\Lambda_{t}\left(\frac{1}{c_{1}}\|\omega(t)\|_{\infty}\right)\right) \leq \frac{c_{3}}{\|\omega(t)\|_{\infty} \Phi\left(\|\omega(t)\|_{\infty}\right)},
$$

where, in our first scenario, $\Phi(x)=\log (1+x)$, and, in the second, $\Phi(x)=\log \log (e+x)$. These results are given in Section 3 . We note for clarity that ours are not stated as regularity criteria but instead scenarios under which 3D NSE becomes sub-critical with regard to the scaling described in [8, 13].

The above decay rates will be obtained by imposing certain structural requirements on the blow-up rates exhibited by vorticity components. Let $\omega_{j}^{+}$and $\omega_{j}^{-}$respectively denote the positive and negative vorticity components truncated away from zero. We will define amenable blow-up rates of orders 0 and 1 in detail in Definition 6 and here only illustrate a class of functions exhibiting such blow-up profiles and describe how the blow-up rates are connected to $\omega_{j}^{ \pm}$. A function $g$ (to be identified with one of the $\omega_{j}^{ \pm} \mathrm{s}$ ) exhibits a local algebraic blow-up (around $x_{0}$, at time $T$ ) if there exists a constant $C>1$ so that, for $(x, t)$ in a parabolic cylinder, $Q=B\left(x_{0}, r\right) \times(0, T]$, we have,

$$
\frac{1}{C}\left(\frac{1}{|p(x, t)|+\tau(t)}\right)^{\alpha(t)} \leq|g(x, t)| \leq C\left(\frac{1}{|p(x, t)|+\tau(t)}\right)^{\alpha(t)}
$$

where, at each time $t, p(\cdot, t)$ is some polynomial of degree less than a fixed natural number $d$, $\alpha$ is positive valued and bounded away from both 0 and $\infty$, and $\tau$ is a positive (up to $T$ ) scalar function of time which vanishes at the singular time. The envisioned blow-up occurs at the zeros of $p(x, T)$ lying within $B\left(x_{0}, r\right)$. There is a considerable amount of freedom present in the above construction as the polynomial is allowed to vary wildly in the time dimension. We will also consider a scenario where some asymmetry is allowed between the bounds assumed on vorticity components. In particular, for appropriate blow-up rates $D_{j}^{ \pm}(x, t)$, we will require,

$$
D_{j}^{ \pm}(x, t) \leq\left|\omega_{j}^{ \pm}(x, t)\right| \leq C D_{j}^{ \pm}(x, t)^{\beta_{j}^{ \pm}(t)},
$$

where $\beta_{j}^{ \pm}:(0, T) \rightarrow\left[1, B_{j}\right]$ for some fixed value $B_{j}$.

A key role is played in our analysis by the cancellations evident in the vortex stretching term in the context of the real Hardy space $\mathcal{H}^{1}$ exploited via the Div-Curl lemma [5] and the $\mathcal{H}^{1}-B M O$ duality [11, 10]. In the standard way this gives uniform-in-time control of the vortex stretching term. The structural blow-up assumptions are provided to ensure uniform-in-time control of the $B M O$ norm of a multiplier (multiplied against the vortex stretching term). In the algebraic case, this is enabled by the logarithm's depletive effect 
on the unboundedness of the mean oscillations of polynomial functions. The effectiveness is witnessed by the remarkable fact (cf. a proof by Stein in [21]) that there exists a constant $C(d, n)$ so that, for any polynomial on $\mathbb{R}^{n}$ of degree less than or equal to $d$,

$$
\|\log |P|\|_{B M O} \leq C(d, n) .
$$

In particular, the constant is independent of the coefficients. This allows us to introduce time-dependent algebraic comparability conditions on the spatial profiles prior to a possibly singular time and do so in a manner that preserves time-independent estimates on the BMO norms of the logarithms of these profiles.

We proceed in Section 2 to review needed results from harmonic analysis and then define the classes of blow-up scenarios which will be amenable to our PDE argument. We also include results which connect the structural blow-up assumptions to an energy inequality-type argument given in Section 3 The statements and proofs of the main results are contained in Section 3 .

\section{Preliminaries and Amenable Blow-Up Rates}

Here we review needed results from harmonic analysis and present a lemma which will connect these ideas to the PDE context of Section 3 . Following [22], the maximal function of a distribution $f$ is defined for all $x \in \mathbb{R}^{n}$ as,

$$
M_{h} f(x)=\sup _{t>0}\left|f * h_{t}(x)\right|,
$$

where $h$ is a fixed test function supported on the unit ball so that $\int h d x=1$ and $h_{t}$ denotes $t^{-n} h(x / t)$.

Definition 1. The distribution $f$ is in the Hardy space $\mathcal{H}^{1}$ if $\|f\|_{\mathcal{H}^{1}}:=\left\|M_{h} f\right\|_{1}<\infty$.

In [5], Coifman, Lions, Meyer, and Semmes reformulated and refined some key features of the 'sequential' theory of compensated compactness within the framework of Hardy spaces, the key idea being that certain nonlinear quantities exhibit cancelations yielding the improved regularity. One such result is the Div-Curl lemma.

Lemma 2. (Coifmann, Lions, Meyer, Semmes - [5]) Suppose $E, B \in L^{2}\left(\mathbb{R}^{3}\right)^{3}$ with $\nabla \cdot E=0$ and $\nabla \times B=0$ (in the sense of distributions). Then, $E \cdot B \in \mathcal{H}^{1}$, and there exists a universal constant $C$ such that

$$
\|E \cdot B\|_{\mathcal{H}^{1}} \leq C\|E\|_{L^{2}}\|B\|_{L^{2}} .
$$

Because weak solutions to the Navier-Stokes equations are divergence free and because the curl of a gradient is always equal to the trivial distribution, it follows that the advective term, $(u \cdot \nabla) u$, in the velocitypressure formulation exhibits div-curl structure, as does the vortex stretching term, $(\omega \cdot \nabla) u$, in the vorticityvelocity formulation. Consequently, using Hardy spaces, refined regularity results can be established for weak solutions of 3D NSE (see Chapter 3.2 of [18] for a collection of such results).

The well known result of Fefferman (cf. [10, 11], as well as Stein's monograph [22]) establishes that the dual space of $\mathcal{H}^{1}$ is precisely the space of functions of bounded mean oscillation, $B M O$. By $f_{B}$ we denote the average of the locally integrable function $f$ over the ball $B$ of volume $|B|$; i.e., $f_{B}=|B|^{-1} \int_{B} f d x$. The mean oscillation of $f$ over $B$ is $|B|^{-1} \int\left|f-f_{B}\right| d x$ and is the quantity typically used to characterize $B M O$.

Definition 3. The locally integrable function $f$ is in $B M O$ if $\|f\|_{B M O}<\infty$ where

$$
\|f\|_{B M O}:=\sup _{x \in \mathbb{R}^{3} ; 0<r} \frac{1}{|B(x, r)|} \int_{B(x, r)}\left|f(y)-f_{B(x, r)}\right| d y .
$$

Several results about the space $B M O$ will expedite future work. An elementary fact is that, if $g \in B M O$, then the following implication holds,

$$
g(x)+m \leq f(x) \leq g(x)+M \text { for a. e. } x \in \mathbb{R}^{3} \quad \Longrightarrow \quad\|f\|_{B M O} \leq\|g\|_{B M O}+M-m .
$$

The above is easy to see by directly comparing $\left|f-f_{B}\right|$ to $\left|g+M-(g+m)_{B}\right|$ and concluding that, for all balls $B$, we have,

$$
\frac{1}{|B|} \int_{B}\left|f-f_{B}\right| d x \leq \frac{1}{|B|} \int_{B}\left|g-g_{B}\right| d x+M-m .
$$


We include a technical lemma regarding certain point-wise multipliers on $B M O$ which appears in a paper by Iwaniec and Verde (cf. Lemma 2.1 of [16]; see also Lemma 5.10 of [2]). This result will allow us to use the standard $\mathcal{H}^{1}-B M O$ duality in an essentially local context instead of working in the local versions of these spaces. It is worth mentioning that our results can be achieved in the local spaces using a local nonhomogeneous version of the Div-Curl lemma (cf. [5]) paired with various local $h^{1}-b m o$ dualities (cf. [2]) thereby obtaining estimates on the localized vortex stretching term (this approach is similar to the estimates in [14]).

Lemma 4. (Iwaniec and Verde - [16]) Suppose $h \in B M O\left(\mathbb{R}^{n}\right)$ and $\phi \in C_{0}^{1}\left(\mathbb{R}^{n}\right)$ is supported on the ball $B$. Then $\phi h \in B M O\left(\mathbb{R}^{n}\right)$ and we have,

$$
\|\phi h\|_{B M O} \leq C(n, R)\|\nabla \phi\|_{\infty}\left(\left(\|h\|_{B M O}+\left|\frac{1}{|B|} \int_{B} h d x\right|\right) .\right.
$$

The space $B M O$ enjoys an intimate connection with the logarithm. This is illustrated in the following lemma due to Stein.

Lemma 5. (Stein - [21]) Let $P$ be any polynomial in $\mathbb{R}^{n}$ of degree less than or equal to $d$. Then there exists a constant $C=C(d, n)$ so that $\log |P| \in B M O$ and

$$
\|\log |P|\|_{B M O} \leq C(d, n) .
$$

The fact that the constant appearing above is independent of the coefficients evidences the remarkable extent to which the logarithm depletes mean oscillations and motivates the assumptions on which the conclusions of Section 3 are conditioned. This will be done by assuming that solutions blow-up in a fashion possessing certain structure. The remainder of this section is dedicated to describing amenable blow-up structures and the statement and proof of a lemma which will connect this structure to the PDE context of 3 . We denote by $\log ^{m}(x)$ an iterated composition of the logarithm with itself $m-1$ times, that is, $\log ^{m}(x)=\log \circ \cdots \circ$ $\log \left(\sqrt{e_{m}+x^{2}}\right)$ where $m$ applications the logarithm are carried out and $e_{m}$ is a power of $e$ defined so that $\log ^{m}(0)=0$. We will use the following notation when defining functions of $(x, t)$ possessing singularities at time $T$ in the spatial set $S \subset \mathbb{R}^{3}$,

$$
Q_{T}\left(x_{0}, R, S\right)=B\left(x_{0}, R\right) \times(0, T) \cup\left(\left(B\left(x_{0}, R\right) \backslash S\right) \times\{T\} \text { and } \Omega_{T}(S)=\mathbb{R}^{3} \times(0, T) \cup\left(\mathbb{R}^{3} \backslash S\right) \times\{T\},\right.
$$

where $R>0$ and $x_{0} \in \mathbb{R}^{3}$. In contexts where $x_{0}$ and $R$ are fixed we will make the abbreviation $Q_{T}(S)=$ $Q_{T}\left(x_{0}, R, S\right)$.

Definition 6. Fix $x_{0} \in \mathbb{R}^{3}, R, T>0, m \in \mathbb{N}_{0}$ and a set of measure zero, $S$, which is contained in a compact subset of $B\left(x_{0}, R\right)$.

a. An amenable blow-up rate of order $m$ on $Q_{T}(S)$ is a function, $D(x, t): \Omega_{T}(S) \rightarrow[0, \infty)$, which additionally satisfies,

i. there exists $M_{0}>0$ so that $\sup _{0<t \leq T}\left\|\log ^{m+1} D(x, t)\right\|_{B M O}<M_{0}$,

ii. there exist $M_{1}, M_{2}>0$ so that $1 / M_{1} \leq D(x, t)$ on $Q_{T}(S)$ and $D(x, t) \leq M_{2}$ on $B\left(x_{0}, R\right)^{c} \times(0, T]$.

b. A function $f(x, t): Q_{T}(S) \rightarrow \mathbb{R}$ exhibits an amenable blow-up rate of order 0 on $Q_{T}(S)$ if there exists an amenable blow-up rate of order $0, D(x, t)$, on $Q_{T}(S)$, so that, for some $C_{*}>1$,

$$
D(x, t) \leq|f(x, t)| \leq C_{*} D(x, t) \text { for all }(x, t) \in Q_{T}(S) .
$$

c. A function $f(x, t): Q_{T}(S) \rightarrow \mathbb{R}$ exhibits an amenable blow-up rate of order 1 on $Q_{T}(S)$ if there exists an amenable blow-up rate of order $1, D(x, t)$, on $Q_{T}(S)$, so that, for some $C_{*}>0, A \geq 1$, and a scalar function $\alpha:[0, T] \rightarrow[1, A]$,

$$
D(x, t) \leq|f(x, t)| \leq C_{*} D(x, t)^{\alpha(t)} \quad \text { for all }(x, t) \in Q_{T}(S) .
$$

The condition (6) a.i) will prove crucial in establishing our estimates in Section 3 At face value, however, it is not clearly motivated. To address this we specify an expansive class of functions which are simultaneously amenable blow-up rates of order 0 and are reasonable blow-up scenarios given what is known about the 
structure of possible singularities in weak solutions of 3D NSE. In the following, $\mathbb{R}\left[x_{1}, x_{2}, x_{3}\right]$ denotes the ring of tri-variate polynomials with coefficients in $\mathbb{R}$.

Definition 7. Use the notations of Definition 6 and fix $d \in \mathbb{N}$. The function $D(x, t): \Omega_{T}(S) \rightarrow[0, \infty)$ is an algebraic blow-up rate of degree $d$ on $Q_{T}(S)$ if there exist functions $\tau:(0, T] \rightarrow[0, \infty), \alpha:(0, T] \rightarrow(0, \infty)$, and $\rho(x, \cdot):(0, T] \rightarrow \mathbb{R}\left[x_{1}, x_{2}, x_{3}\right]$ so that,

$$
D(x, t)=\left(\frac{1}{|\rho(x, t)|+\tau(t)}\right)^{\alpha(t)} \text { on } Q_{T}(S),
$$

and we additionally have,

i. for all $t \in(0, T], \rho(x, t)$ is a polynomial of degree less than or equal to $d$ and the zeros of $\rho(x, T)$ are contained in $S$,

ii. there exists $A \geq 1$ so that $\alpha$ takes values in $\left[A^{-1}, A\right]$,

iii. $\tau(t)>0$ for $t \in(0, T)$ and vanishes as $t$ approaches $T$,

iv. the condition (6)a.ii.) is satisfied.

Remark 8. We have presented these algebraic blow-up rates because they constitute a concrete class of amenable blow-up rates of order 0 . To verify this, we check that condition (6)a.i.) is satisfied, which is clear if we first expand $\log D(x, t)$ as,

$$
\log (D(x, t))=-\alpha(t) \log (|\rho(x, t)|+\tau(t)),
$$

and, additionally observe that,

$$
\max \{\log |\rho(x, t)|, \log \tau(t)\} \leq \log (|\rho(x, t)|+\tau(t)) \leq \log (2)+\max \{\log |\rho(x, t)|, \log \tau(t)\} .
$$

Recalling the fact that, if $f, g \in B M O$, then $\|\max \{f, g\}\|_{B M O} \leq 2\left(\|f\|_{B M O}+\|g\|_{B M O}\right)(B M O$ is a lattice), we are able to conclude by applying the implication (1) in conjunction with Lemma 5 .

Our main lemma contextualizes amenable blow-up rates to their application in Section 3 As presently defined, the blow-up rates require that $f(x, t)$ (as given in Definition 6) is bounded away from zero. To accommodate functions possibly not bounded away from zero we employ an auxiliary function, $F=\sqrt{e_{m}+f^{2}}$, where $e_{m}$ is determined by the order of the amenable blow-up rate in question. Based on the sign of $f$ we define,

$$
F_{+}=\left\{\begin{array}{ll}
F, & \text { if } f \text { is non-negative, } \\
1, & \text { otherwise, }
\end{array} \quad F_{-}= \begin{cases}1, & \text { if } f \text { is non-negative, } \\
F, & \text { otherwise. }\end{cases}\right.
$$

The factorization $F^{\operatorname{sign} f}=F_{+}\left(F_{-}\right)^{-1}$ will allow us to independently impose blow-up assumptions on a function's positive and negative components.

Lemma 9. Fix $R_{0}>0$. Let $S^{\prime} \subset \mathbb{R}^{3}$ be a set of Lebesque measure zero for which $S^{\prime} \cap B\left(0, R_{0}\right) \subset B\left(0, R_{0}^{\prime}\right)$ where $0<R_{0}^{\prime}<R_{0}$. Suppose the function $f: \Omega_{T}\left(S^{\prime}\right) \rightarrow \mathbb{R}$ satisfies $f \in L^{\infty}\left((0, T] ; L^{1}\left(B\left(0, R_{0}\right)\right)\right)$. Fix $\phi \in C_{0}^{1}\left(\mathbb{R}^{3}\right)$ so that $\phi$ is supported on $B(0, R)$ and let $S$ be a set of measure zero satisfying $S^{\prime} \cap B\left(0, R_{0}\right) \subset S \subset B\left(0, R_{0}\right)$.

a. If $\left.f\right|_{Q_{T}(S)}$ exhibits an amenable blow-up of order 0 in $Q_{T}(S)$, then,

$$
\sup _{0<t \leq T}\|\phi(x) \log \mid f(x, t)\| \|_{B M O}<\infty .
$$

b. If $\left.F_{+}\right|_{Q_{T}(S)}$ and $\left.F_{-}\right|_{Q_{T}(S)}$ exhibit amenable blow-up rates of order 0 in $Q_{T}(S)$, then,

$$
\sup _{0<t \leq T}\left\|\phi(x) \frac{f(x, t)}{F(x, t)} \log F(x, t)\right\|_{B M O}<\infty .
$$

c. If $\left.F_{+}\right|_{Q_{T}(S)}$ and $\left.F_{-}\right|_{Q_{T}(S)}$ exhibit amenable blow-up rates of order 1 in $Q_{T}(S)$, then,

$$
\sup _{0<t \leq T}\left\|\phi(x) \frac{f(x, t)}{F(x, t)} \log \log F(x, t)\right\|_{B M O}<\infty .
$$


Proof. (Part a.) Let $D(x, t)$ be the amenable blow-up rate exhibited by $\left.f\right|_{Q_{T}(S)}$. We will freely reference the constants associated with $D$ in the statement of Definition 6 and subsequently make the abbreviations $Q_{T}(S)=Q_{T}\left(0, R_{0}, S\right)$ and $B=B\left(0, R_{0}\right)$. Define an extension, $\widetilde{F}$, of $\left.f\right|_{Q_{T}(S)}$ to $\Omega_{T}(S)$ by $\widetilde{F}=|f|$ on $Q_{T}(S)$ and $\widetilde{F}=1$ on $B^{c} \times(0, T]$. Set $\widetilde{D}(x, t)=\max \left\{D(x, t), M_{1}^{-1}\right\}$ and $\widetilde{C}_{*}=\max \left\{C_{*}, M_{2}\right\}$. These definitions ensure that, for all $(x, t) \in \Omega_{T}(S)$,

$$
\frac{1}{\widetilde{C}_{*}} \widetilde{D}(x, t) \leq \widetilde{F}(x, t) \leq \widetilde{C}_{*} \widetilde{D}(x, t),
$$

and, taking logarithms,

$$
\log (\widetilde{D}(x, t))-\log \widetilde{C}_{*} \leq \log (\widetilde{F}(x, t)) \leq \log (\widetilde{D}(x, t))+\log \widetilde{C}_{*} .
$$

Recalling the implication (1), we see that,

$$
\|\log \widetilde{F}(x, t)\|_{B M O} \leq C\left(M_{0}, \tilde{C}_{*}\right) .
$$

Because $|f|=\widetilde{F}$ on the support of $\phi$ we have,

$$
\begin{aligned}
\|\phi \log |f|\|_{B M O} & =\|\phi \log \widetilde{F}\|_{B M O} \\
& \leq C\|\nabla \phi\|_{\infty}\left(\|\log \widetilde{F}\|_{B M O}+\left|\frac{1}{|B|} \int_{B} \log \widetilde{F} d x\right|\right),
\end{aligned}
$$

where we have applied Lemma 4 . The average appearing above is uniformly bounded in time; this follows from the facts that $M_{1}^{-1} \leq|f|$ and $f \in L^{\infty}\left((0, T] ; L^{1}\left(B\left(0, R_{0}\right)\right)\right)$.

(Part b.) We begin by remarking that, for all $y \in \mathbb{R}$,

$$
\left.(\operatorname{sign} y) \log \left(\sqrt{1+y^{2}}\right)-1 \leq \frac{y}{\sqrt{1+y^{2}}} \log \left(\sqrt{1+y^{2}}\right) \leq(\operatorname{sign} y) \log \sqrt{1+y^{2}}\right)+1 .
$$

Taking $y=|f(x, t)|$, in light of implication (1) it is sufficient to work with the function ( $\operatorname{sign} f) \log F$, where $F$, and also $F_{+}$and $F_{-}$, are defined as in the comments preceding the statement of the lemma. Those definitions were motivated by the fact that $F^{\operatorname{sign} f}=F_{+}\left(F_{-}\right)^{-1}$ and, so,

$$
\log \left(F^{\operatorname{sign} f}\right)=\log \left(F_{+}\right)-\log \left(F_{-}\right) .
$$

Let $G$ be the extension by 1 of $\left.\left(f F^{-1} \log (F)\right)\right|_{Q_{T}(S)}$ from $Q_{T}(S)$ to $\Omega_{T}(S)$ and adopt the notation from the proof of part a. of this lemma for definitions of functions analogous to those mentioned above where we designate by $D_{ \pm}$the blow-up rates exhibited by $F_{ \pm}$. Then, on $\Omega_{T}(S)$, we have,

$$
\log \left(\widetilde{F}_{+}\right)-\log \left(\widetilde{F}_{-}\right)-1 \leq G \leq \log \left(\widetilde{F}_{+}\right)-\log \left(\widetilde{F}_{-}\right)+1,
$$

and, therefore,

$$
\|G\|_{B M O} \leq\left\|\log \left(\widetilde{F}_{+}\right)\right\|_{B M O}+\left\|\log \left(\widetilde{F}_{-}\right)\right\|_{B M O}+2 .
$$

Appealing to part a. of the lemma, the dominating quantity is itself bounded uniformly in time. Extending this to the desired estimate proceeds directly,

$$
\left\|\phi \frac{f}{F} \log (F)\right\|_{B M O} \leq C\|\nabla \phi\|_{\infty}\left(\|G\|_{B M O}+\left|\frac{1}{B} \int_{B} \log \widetilde{G} d x\right|\right),
$$

which, again, is finite by assumptions on $f$.

(Part c.) We begin similarly, noting,

$$
(\operatorname{sign} f) \log \log F-1 \leq \frac{f}{F} \log \log F \leq(\operatorname{sign} f) \log \log F+1 .
$$

This leads us to consider,

$$
\log \left((\log F)^{\operatorname{sign} f}\right)=\log \left((\log F)_{+}\right)+\log \left((\log F)_{-}\right),
$$


where,

$$
(\log F)_{+}=\max \left\{(\log F)^{\operatorname{sign} f}, 1\right\} \quad \text { and } \quad(\log F)_{-}=\min \left\{(\log F)^{\operatorname{sign} f}, 1\right\} .
$$

It is easy to check that $(\log F)_{ \pm}=\log \left(F_{ \pm}\right)$, and, therefore,

$$
\frac{f}{F} \log \log F=\log \log \left(F_{+}\right)-\log \log \left(F_{-}\right) .
$$

A further observation is that, taking $D_{ \pm}(x, t)$ to be the amenable blow-up rates exhibited by $F_{ \pm}$, and setting $k_{ \pm}^{*}=\log \left(A_{ \pm} M_{1, \pm}+\log C_{*, \pm}\right)$ (these are constants associated with $D_{ \pm}$as in Definition 6 a.), we have for $(x, t) \in Q_{T}$,

$$
\log \log \left(C_{*} D_{ \pm}(x, t)^{\alpha_{ \pm}(t)}\right) \leq \log \log \left(D_{ \pm}(x, t)\right)+k_{ \pm}^{*},
$$

and, recalling the comparability condition from Definition 6 c., this implies that,

$$
\log \log \left(D_{ \pm}(x, t)\right) \leq F_{ \pm} \leq \log \log \left(D_{ \pm}(x, t)\right)+k_{ \pm}^{*} .
$$

The conclusion now follows in the same fashion laid out in part b.; we omit the details.

\section{MAin Results}

Weak solutions to the 3D incompressible Navier-Stokes equations are functions which satisfy (distributionally) the following system of PDEs,

$$
\left\{\begin{array}{l}
\partial_{t} u+(u \cdot \nabla) u=-\nabla p+\nu \Delta u, \\
\nabla \cdot u=0 ; \quad u(x, 0)=u_{0} \in H,
\end{array}\right.
$$

where $H$ is the $L^{2}$ closure of the divergence free test functions and the initial datum is understood in the sense of weak continuity (cf. [7] for details). The evolution of the vorticity, $\omega=\nabla \times u$, is of special interest to us and satisfies,

$$
\left\{\begin{array}{c}
\partial_{t} \omega+(u \cdot \nabla) \omega=(\omega \cdot \nabla)+\nu \Delta \omega \\
\nabla \cdot \omega=0 .
\end{array}\right.
$$

For simplicity, we consider a weak solution on $\mathbb{R}^{3} \times(0, \infty)$, evolving from the initial data $u_{0}$. We also require that the initial vorticity, $\omega_{0}=\nabla \times u_{0}$, is in $L^{1} \cap L^{2}$.

The standard regularity results for weak solutions (cf. [7]) consist of the a priori bounds,

$$
\sup _{0<t<T^{\prime}}\|u\|_{L^{2}\left(\mathbb{R}^{3}\right)}^{2}<\infty \text { and } \int_{0}^{T^{\prime}}\|\nabla u\|_{L^{2}\left(\mathbb{R}^{3}\right)}^{2} d t<\infty,
$$

for any $T^{\prime}>0$. In addition, since $\omega_{0} \in L^{1}$, a result from [6] ensures that,

$$
\sup _{0 \leq t \leq T^{\prime}}\|\omega\|_{L^{1}\left(\mathbb{R}^{3}\right)}<\infty .
$$

Note that since $\omega_{0} \in L^{2}$, our weak solution locally-in-time coincides with the smooth solution; let $T$ be the first (possible) singular time.

Fix a 'macro-scale,' $R_{0}>0$, with the property that the intersection of $B\left(0, R_{0}\right) \times\{T\}$ with the singular set at time $T$ is nonempty. Fix $0<\epsilon<R_{0}$. Our estimates are intended for integrals over the spatial set $B\left(0, R_{0}-\epsilon\right)$ and localization is achieved via multiplying equation (4) by a smooth cut-off function $\psi$ satisfying,

$$
\operatorname{supp} \psi \subset B\left(0, R_{0}\right), \quad \psi=1 \text { on } B\left(0, R_{0}-\epsilon\right), \quad \frac{|\nabla \psi|}{\psi^{\rho}} \leq \frac{c}{\epsilon} \text { for some } \rho \in(0,1) \quad \text { and } \quad 0 \leq \psi \leq 1 .
$$

Instead of studying the evolution of $|\omega|$ and $\left|\omega_{k}\right|$ directly we introduce an auxiliary function. This approach is an adaptation of that taken by Constantin in [6]. Define $q(y)=\sqrt{1+y^{2}}: \mathbb{R} \rightarrow \mathbb{R}$ and let $w_{k}=q\left(\omega_{k}\right)$ : $\mathbb{R}^{3} \rightarrow \mathbb{R}$. From these definitions it is immediate that,

$$
\left|\omega_{k}\right| \leq w_{k}, \quad-1 \leq q^{\prime}\left(\omega_{k}\right)=\frac{\omega_{k}}{w_{k}} \leq 1, \quad \text { and } \quad 0<q^{\prime \prime}\left(\omega_{k}\right)=\frac{1}{w_{k}^{3}} \leq 1 .
$$


For convenience we recall the following elementary facts (for $y \geq 1$ ),

$$
0 \leq-\log ^{\prime \prime}(y) \leq \log ^{\prime}(y) \leq 1, \quad y \log ^{\prime} y=1, \quad \text { and } \quad 0=\log ^{\prime}(y)+y \log ^{\prime \prime}(y)
$$

To allow $\omega_{k}$ to have varying sign we define the functions $w_{k,+}$ and $w_{k,-}$ in the same manner as (2). For convenience we recall the notation $\Lambda_{t}(y)=\{x:|\omega(x, t)| \geq y\}$. Also, throughout the remainder of this paper, $c_{1}$ denotes a fixed constant taken to be greater than one. The role of $c_{1}$ lies in specifying the threshold for the super-level sets $\Lambda_{t}(y)$ as fractions of the supremum norm of the modulus of the vorticity at the time $t$, i.e. $y=c_{1}^{-1}\|\omega(\cdot, t)\|_{\infty}$.

We include two theorems, one each for the case of solutions possessing vorticity components which exhibit amenable blow-up rates of order 0 and of order 1 . The proof of the order 0 case extends easily to the case of blow-ups of order 1 and the proof of the second result is accordingly terse.

Theorem 10. Let $u$ be a Leray solution to (3) on $\mathbb{R}^{3} \times(0, \infty)$, and suppose additionally that $\omega_{0}=\nabla \times u_{0} \in L^{1} \cap L^{2}$. Denote by $T$ the first singular time and by $S$ the singular set of $\omega$ at time $T$. Fix positive values $R_{0}$ and $\epsilon$ so that $\emptyset \neq S \cap B\left(0, R_{0}\right) \subset B\left(0, R_{0}-\epsilon\right)$ - i.e. there are singular points in $B\left(0, R_{0}\right)$ but all such points are in $B\left(0, R_{0}-\epsilon\right)$ - and let $Q_{T}(S)=Q_{T}\left(0, R_{0}, S\right)$.

(i) If $\left.w_{k,+}\right|_{Q_{T}(S)}$ and $\left.w_{k,-}\right|_{Q_{T}(S)}$ each exhibit amenable blow-up rates of order 0 in $Q_{T}(S)$, then there exists a positive value $M_{k}$ for which,

$$
\sup _{t \in[0, T]} \int_{B\left(0, R_{0}-\epsilon\right)}\left|\omega_{k}(x, t)\right| \log \left(\sqrt{1+\left|\omega_{k}(x, t)\right|^{2}}\right) d x<M_{k} .
$$

(ii) Let $c_{1}$ ba a fixed constant which is greater than 1 . If, for all $k$, the premises of part (i) are satisfied, then there exists a positive constant $M_{0}$ so that, for $t \in[0, T]$,

$$
\operatorname{Vol}\left(\Lambda_{t}\left(\frac{1}{c_{1}}\|\omega(t)\|_{L^{\infty}\left(B\left(0, R_{0}-\epsilon\right)\right)}\right)\right) \leq \frac{M_{0}}{\|\omega(t)\|_{L^{\infty}\left(B\left(0, R_{0}-\epsilon\right)\right)} \log \left(1+\|\omega(t)\|_{L^{\infty}\left(B\left(0, R_{0}-\epsilon\right)\right)}\right)} .
$$

Remark 11. As will be seen, our energy inequality-type method depends heavily on classical techniques and we thus need to consider smooth solutions. It is possible to extend the estimates to some weak solutions by considering a sequence of smooth approximations - a good choice to use here is the method of retarded mollifiers given for suitable weak solutions in [1] as it allows one to recover from a velocity-level approximation scheme information about the vorticity, see [6] for more discussion - but, as our estimates hinge on assumptions beyond the initial data, $u_{0}$, we would have to ensure these are met by a convergent sub-sequence of approximate solutions.

Proof. In virtue of our smoothness assumption, the evolution of $\psi w_{k} \log \left(w_{k}\right)$ can be established from the evolution of $\omega_{k}$ by first writing,

$$
\begin{aligned}
& \partial_{t}\left(\psi w_{k} \log w_{k}\right) \\
& =\psi q^{\prime}\left(\omega_{k}\right)\left(\log w_{k}+w_{k} \log ^{\prime} w_{k}\right)\left(\partial_{t} \omega_{k}\right) \\
& =\psi q^{\prime}\left(\omega_{k}\right)\left(\log w_{k}+w_{k} \log ^{\prime} w_{k}\right)\left(\nu \Delta \omega_{k}-(u \cdot \nabla) \omega_{k}+(\omega \cdot \nabla) u_{k}\right),
\end{aligned}
$$

and then deriving (noting tacit summation over terms involving indices other than $k$ ),

$$
\begin{aligned}
& \partial_{t}\left(\psi w_{k} \log w_{k}\right)-\nu \psi \Delta w_{k}\left(\log w_{k}+w_{k} \log ^{\prime} w_{k}\right)+\nu \psi q^{\prime \prime}\left(\omega_{k}\right)\left(\partial_{i} \omega_{k}\right)^{2}\left(\log w_{k}+w_{k} \log ^{\prime} w_{k}\right) \\
& =\psi(\omega \cdot \nabla) u_{k} q^{\prime}\left(\omega_{k}\right)\left(\log w_{k}+w_{k} \log ^{\prime} w_{k}\right)-\psi(u \cdot \nabla) w_{k}\left(\log w_{k}+w_{k} \log ^{\prime} w_{k}\right) .
\end{aligned}
$$


By integrating in space and time and dropping the positive quantity involving $q^{\prime \prime}$ from the left hand side, we obtain the following energy inequality-type estimate,

$$
\begin{aligned}
& \int \psi(t) w_{k}(t) \log w_{k}(t) d x-\nu \int_{0}^{t} \int \psi \Delta w_{k}\left(\log w_{k}+w_{k} \log ^{\prime} w_{k}\right) d x d s \\
& \leq \int_{0}^{t} \int \psi(\omega \cdot \nabla) u_{k} q^{\prime}\left(\omega_{k}\right)\left(\log w_{k}+w_{k} \log ^{\prime} w_{k}\right) d x d s \\
& \quad-\int_{0}^{t} \int \psi(u \cdot \nabla) w_{k}\left(\log w_{k}+w_{k} \log ^{\prime} w_{k}\right) d x d s \\
& \quad+\int \psi w_{k, 0} \log w_{k, 0} d x .
\end{aligned}
$$

The properties in (6) enable several key cancellations. For the dissipative terms, integration by parts reveals that,

$$
\begin{aligned}
\int_{0}^{t} \int\left(\partial_{j}^{2} w_{k}\right) \psi \log w_{k} d x d s= & \int_{0}^{t} \int\left(w_{k}\left(\partial_{j}^{2} \psi\right) \log w_{k}+w_{k}\left(\partial_{j} \psi\right)\left(\partial_{j} w_{k}\right) \log ^{\prime} w_{k}\right) d x d s \\
& -\int_{0}^{t} \int\left(\partial_{j} w_{k}\right)^{2} \psi \log ^{\prime} w_{k} d x d s
\end{aligned}
$$

and,

$$
\begin{aligned}
\int_{0}^{t} \int\left(\partial_{j}^{2} w_{k}\right) \psi w_{k} \log ^{\prime} w_{k} d x d s= & -\int_{0}^{t} \int w_{k}\left(\partial_{j} \psi\right)\left(\partial_{j} w_{k}\right) \log ^{\prime} w_{k} d x d s \\
& -\int_{0}^{t} \int\left(\partial_{j} w_{k}\right)^{2} \psi\left(\log ^{\prime} w_{k}+w_{k} \log ^{\prime \prime} w_{k}\right) d x d s
\end{aligned}
$$

A cancellation occurs upon adding the above equations leaving us with,

$$
\begin{aligned}
& -\nu \int_{0}^{t} \int\left(\partial_{j}^{2} w_{k}\right) \psi\left(\log w_{k}+w_{k} \log ^{\prime} w_{k}\right) d x d s \\
& =\nu \int_{0}^{t} \int\left(\partial_{j} w_{k}\right)^{2} \psi\left(2 \log ^{\prime} w_{k}+w_{k} \log ^{\prime \prime} w_{k}\right) d x d s-\nu \int_{0}^{t} \int w_{k}\left(\partial_{j}^{2} \psi\right) \log w_{k} d x d s .
\end{aligned}
$$

Again noting (6), the integrand of the first term is positive and, therefore, can be dropped from the left hand side of estimate (7). The second term can be dominated by an a priori finite quantity arising from the standard energy inequality for weak solutions in conjunction with the fact that our spatial integral is over a set of finite measure. More precisely,

$$
\begin{aligned}
\int_{0}^{t} \int w_{k}\left(\partial_{j}^{2} \psi\right) \log w_{k} d x d s & \leq C \int_{0}^{T} \int_{B\left(0, R_{0}\right)} w_{k}^{2} d x d s \\
& =C \int_{0}^{T} \int_{B\left(0, R_{0}\right)}\left|\omega_{k}\right|^{2} d x d s+T\left|B\left(0, R_{0}\right)\right| .
\end{aligned}
$$

The integrals arising from the transport term in (4) also enjoy substantial cancellations,

$$
\begin{aligned}
& \int_{0}^{t} \int \psi u \cdot \nabla w_{k}\left(\log w_{k}+w_{k} \log ^{\prime} w_{k}\right) d x d s \\
& =\int_{0}^{t} \int \psi u_{j}\left(\partial_{j} w_{k}\right) w_{k} \log ^{\prime} w_{k} d x d s-\int_{0}^{t} \int \psi u_{j} w_{k}\left(\partial_{j} w_{k}\right) \log ^{\prime} w_{k} d x d s \\
& \quad-\int_{0}^{t} \int u_{j} w_{k} \log w_{k} \partial_{j} \psi d x d s \\
& =-\int_{0}^{t} \int u_{j} w_{k} \log w_{k} \partial_{j} \psi d x d s .
\end{aligned}
$$


Noting that $\log (y) \leq 4 y^{1 / 4}$,

$$
\begin{aligned}
& \left|\int_{0}^{t} \int u_{j} w_{k} \log w_{k}\right| \partial_{j} \psi|d x d s| \\
& \leq C \int_{0}^{T} \int\left(\left|\partial_{j} \psi\right|^{1 / 4}\left|u_{j}\right|\right)\left(\left|\partial_{j} \psi\right|^{1 / 2}\left|w_{k}\right|\right)\left|w_{k} \partial_{j} \psi\right|^{1 / 4} d x d s \\
& \leq C \int_{0}^{T}\left\|\psi^{\rho / 4} u\right\|_{4}\left\|w_{k}\right\|_{L^{1}\left(B\left(0, R_{0}\right)\right)}^{1 / 4}|| w_{k} \|_{L^{2}\left(B\left(0, R_{0}\right)\right)} \\
& \leq C \sup _{0<t \leq T}\left\|w_{k}\right\|_{L^{1}\left(B\left(0, R_{0}\right)\right)}^{1 / 4} \int_{0}^{T}\left\|\nabla\left(\psi^{\rho / 4} u\right)\right\|_{2}\left\|w_{k}\right\|_{L^{2}\left(B\left(0, R_{0}\right)\right)},
\end{aligned}
$$

where we have used Hölder's inequality and the Sobolev inequality. From here and in light of (8), a commutator estimate on the gradient of the localized velocity allows the extension of the above estimate to one in terms of a priori finite quantities.

The integral on the last line of the right hand side of (7) is finite by our assumptions on the initial data.

At this point, based on (7), we have established,

$$
\int\left(\psi w_{k} \log w_{k}\right)(t) d x \leq\left|\int_{0}^{t} \int \psi \omega \cdot \nabla u_{k} q^{\prime}\left(\omega_{k}\right)\left(\log w_{k}+w_{k} \log ^{\prime} w_{k}\right) d x d s\right|+R,
$$

where $R$ is comprised of those a priori bounded quantities accumulated in the preceding estimates. Noting that,

$$
\psi q^{\prime}\left(\omega_{k}\right) w_{k} \log ^{\prime} w_{k} \leq 1,
$$

an a priori bound follows for that part of the as of yet unbounded quantity leaving us with,

$$
\int\left(\psi w_{k} \log w_{k}\right)(t) d x \leq\left|\int_{0}^{t} \int \psi \omega \cdot \nabla u_{k} \frac{\omega_{k}}{w_{k}} \log w_{k} d x d s\right|+R .
$$

The $\mathcal{H}^{1}-B M O$ duality and the Div-Curl lemma justify the following chain of inequalities,

$$
\begin{aligned}
& \left|\int_{0}^{t} \int\left(\omega \cdot \nabla u_{k}\right)\left(\psi \frac{\omega_{k}}{w_{k}} \log w_{k}\right) d x d s\right| \\
& \leq \int_{0}^{T}\left\|\omega \cdot \nabla u_{k}\right\|_{\mathcal{H}^{1}} \| \psi \frac{\omega_{k}}{w_{k}} \log w_{k}||_{B M O} d s \\
& \leq\left(\sup _{0<t \leq T}\left\|\psi \frac{\omega_{k}}{w_{k}} \log w_{k}\right\|_{B M O}\right) \int_{0}^{T}\left\|\omega \cdot \nabla u_{k}\right\|_{\mathcal{H}^{1}} d s \\
& \leq\left(\sup _{0<t \leq T}\left\|\psi \frac{\omega_{k}}{w_{k}} \log w_{k}\right\|_{B M O}\right)\left(\int_{0}^{T}\|\omega\|_{2}^{2} d t\right)^{1 / 2}\left(\int_{0}^{T}\|\nabla u\|_{2}^{2} d t\right)^{1 / 2} .
\end{aligned}
$$

By Lemma 9 and the standard regularity of Leray weak solutions all of the above are finite and we have thus established that, for all $0<t \leq T,\left\|\psi w_{k} \log w_{k}(t)\right\|_{L^{1}\left(\mathbb{R}^{3}\right)}$ is majorized by time-independent a priori bounded quantities, the sum of which we label $M_{k}$. This completes our proof of part (i) of the theorem.

Part (ii) of the theorem is proven in two steps. For the first, let,

$$
\lambda(t)=\frac{1}{c_{1}}\|\omega(t)\|_{L^{\infty}\left(B\left(0, R_{0}-\epsilon\right)\right)},
$$

and observe that, for any $x \in B\left(0, R_{0}-\epsilon\right)$ where $|\omega(x, t)| \geq \lambda(t)$, direct computation affirms that,

$$
1 \leq \frac{c_{1}|\omega(x, t)|\left[\log \left(c_{1}\right)+\log (1+|\omega(x, t)|)\right]}{\|\omega(t)\|_{L^{\infty}\left(B\left(0, R_{0}-\epsilon\right)\right)} \log \left(1+|| \omega(t) \|_{L^{\infty}\left(B\left(0, R_{0}-\epsilon\right)\right)}\right)} .
$$


This allows us to estimate the volume of the relevant super-level set of $|\omega|$ at time $t$,

$$
\begin{aligned}
\operatorname{Vol}\left(\Lambda_{t}\left(\frac{1}{c_{1}}\|\omega(t)\|_{L^{\infty}\left(B\left(0, R_{0}-\epsilon\right)\right)}\right)\right) \leq & \frac{c_{1} \log \left(c_{1}\right)\|\omega(t)\|_{L^{1}\left(\{|\omega(x, t)| \geq \lambda(t)\} \cap B\left(0, R_{0}-\epsilon\right)\right)}}{\|\omega(t)\|_{L^{\infty}\left(B\left(0, R_{0}-\epsilon\right)\right)} \log \left(1+\|\omega(t)\|_{L^{\infty}\left(B\left(0, R_{0}-\epsilon\right)\right)}\right)} \\
& +\frac{c_{1}\|\omega(t) \log (1+|\omega(t)|)\|_{L^{1}\left(\{|\omega(x, t)| \geq \lambda(t)\} \cap B\left(0, R_{0}-\epsilon\right)\right)}}{\|\omega(t)\|_{L^{\infty}\left(B\left(0, R_{0}-\epsilon\right)\right)} \log \left(1+\|\omega(t)\|_{L^{\infty}\left(B\left(0, R_{0}-\epsilon\right)\right)}\right)} \\
\leq & \frac{K I_{0}(t)}{\|\omega(t)\|_{L^{\infty}\left(B\left(0, R_{0}-\epsilon\right)\right)} \log \left(1+\|\omega(t)\|_{L^{\infty}\left(B\left(0, R_{0}-\epsilon\right)\right)}\right)},
\end{aligned}
$$

where we have set,

$$
I_{0}(t)=\int_{\{|\omega(x, t)| \geq \lambda(t)\} \cap B\left(0, R_{0}-\epsilon\right)}|\omega(x, t)| \log (1+|\omega(x, t)|) d x,
$$

and have introduced a time-independent constant $K$ which depends on the fixed values $c_{1}$ and $R_{0}$ as well as the $a$ priori finite quantity $\sup _{0 \leq t \leq T}|| \omega(\cdot, t) \|_{L^{1}\left(\{|\omega(x, t)| \geq \lambda(t)\} \cap B\left(0, R_{0}-\epsilon\right)\right)}$.

The second step ensures we can control $I_{0}(t)$ in terms of the finite bounds appearing in part (i) of the theorem. Tacitly summing over $j$, we have,

$$
I_{0}(t) \leq C \int_{B\left(0, R_{0}-\epsilon\right)}\left|\omega_{j}\right| \log \left(1+\sqrt{\omega_{1}^{2}+\omega_{2}^{2}+\omega_{3}^{2}}\right) d x
$$

An explicit reduction illustrates the argument (for simplicity we take $j=1$ and integrals over the indicated sets intersected with $\left.B\left(0, R_{0}-\epsilon\right)\right)$,

$$
\begin{aligned}
& \int\left|\omega_{1}\right| \log \left(1+\sqrt{\omega_{1}^{2}+\omega_{2}^{2}+\omega_{3}^{2}}\right) d x \\
& \leq \int_{\omega_{1}^{2} \leq \omega_{2}^{2}+\omega_{3}^{2}} \sqrt{\omega_{2}^{2}+\omega_{3}^{2}} \log \left(1+\sqrt{2\left(\omega_{2}^{2}+\omega_{3}^{2}\right)}\right) d x+\int_{\omega_{1}^{2}>\omega_{2}^{2}+\omega_{3}^{2}}\left|\omega_{1}\right| \log \left(1+\sqrt{2}\left|\omega_{1}\right|\right) d x .
\end{aligned}
$$

Applying the same reasoning to the first integral above and then repeating for all values of $j$ eventually yields,

$$
I_{0}(t) \leq C \int w_{i} d x+C \int w_{i} \log w_{i} d x \leq C M_{i}
$$

The energy inequality-type construction used to prove the previous theorem also works if we substitute $\log ^{m} \omega_{k}$ (as defined in Section 2) in place of $\log w_{k}$. This allows the application of amenable blow-up rates of order 1 in conjunction with Lemma 9 c. To ensure things are meaningful, we modify our definition of $q$ so that $q(y)=\sqrt{e+y^{2}}$ (so, now, $w_{k}=\sqrt{e+\omega_{k}^{2}}$ ) and refer to (2) to define $w_{k,+}$ and $w_{k,-}$.

Theorem 12. Let $u$ be a Leray solution to (3) on $\mathbb{R}^{3} \times(0, \infty)$, and suppose additionally that $\omega_{0}=\nabla \times u_{0} \in L^{1} \cap L^{2}$. Denote by $T$ the first singular time and by $S$ the singular set of $\omega$ at time $T$. Fix positive values $R_{0}$ and $\epsilon$ so that $\emptyset \neq S \cap B\left(0, R_{0}\right) \subset B\left(0, R_{0}-\epsilon\right)$ - i.e. there are singular points in $B\left(0, R_{0}\right)$ but all such points are in $B\left(0, R_{0}-\epsilon\right)$ - and let $Q_{T}(S)=Q_{T}\left(0, R_{0}, S\right)$.

(i) If $\left.w_{k,+}\right|_{Q_{T}(S)}$ and $\left.w_{k,-}\right|_{Q_{T}(S)}$ each exhibit amenable blow-up rates of order 1 in $Q_{T}(S)$, then there exists a positive value $M_{k}$ so that,

$$
\sup _{t \in[0, T]} \int_{B\left(0, R_{0}-\epsilon\right)}\left|\omega_{k}(x, t)\right| \log \log \left(\sqrt{e+\left|\omega_{k}(x, t)\right|^{2}}\right) d x<M_{k} .
$$

(ii) Let $c_{1}$ ba a fixed constant which is greater than 1 . If, for all $k$, the premises of part (i) are satisfied, then there exists a positive value $M_{0}$ so that, for $t \in[0, T]$,

$$
\operatorname{Vol}\left(\Lambda_{t}\left(\frac{1}{c_{1}}\|\omega(t)\|_{L^{\infty}\left(B\left(0, R_{0}-\epsilon\right)\right)}\right)\right) \leq \frac{M_{0}}{\|\omega(t)\|_{L^{\infty}\left(B\left(0, R_{0}-\epsilon\right)\right)} \log \log \left(e+\|\omega(t)\|_{L^{\infty}\left(B\left(0, R_{0}-\epsilon\right)\right)}\right)} .
$$


Proof. Multiplying equation (4) by $\psi q^{\prime}\left(w_{k}\right)\left(\log \log w_{k}+w_{k}(\log \log )^{\prime} w_{k}\right)$ we obtain the evolution of $\psi w_{k} \log \log w_{k}$. The point-wise estimates in (6) adapt directly to the function $\log \log (y)$ (indeed, they adapt to any number of such self-compositions of the logarithm) and, after integrating in space and time, all of the estimates and cancellations from the previous proof - except those involving the vortex stretching term - can be duplicated directly. We thus obtain, denoting by $R$ some a priori finite quantity,

$$
\int\left(\psi w_{k} \log \log w_{k}\right)(t) d x \leq\left|\int_{0}^{t} \int \psi \omega \cdot \nabla u_{k} \frac{\omega_{k}}{w_{k}} \log \log w_{k} d x d s\right|+R,
$$

and, using Lemma 9 c., we are able to pull out of the integral the uniformly-in-time bounded quantity,

$$
\sup _{t}\left\|\psi \frac{\omega_{k}}{w_{k}} \log \log w_{k}\right\|_{B M O}
$$

and conclude exactly as in the previous proof.

Remark 13. The two theorems apply to any local, spatially-algebraic blow-up scenario in which the degrees of the polynomials stay uniformly bounded near the (possible) singular time. Geometrically, this corresponds to the number of vortex filaments being uniformly bounded as the flow approaches the singular time. This is somewhat unsatisfactory since, a priori, one can not rule out a scenario in which the number of coherent structures runs off to infinity. The technical reason behind this restriction is that the bound on $\|\log |P|\|_{B M O}$ blows up as the degree of $P, d$, goes to infinity.

Fortunately, the bound on $\|\log |P|\|_{B M O}$ is linear in $d$ (cf. [9]). Consequently, although the bound on the distribution function, i.e., on the total volume of the super-level sets will blow up (with $d$ ), at least in the case of comparable volumes, the bound on the volume of a single vortex filament will still be sub-critical (which suffices due to the local nature of the argument).

Acknowledgements. Z.B. acknowledges the support of the Virginia Space Grant Consortium via the Graduate Research Fellowship; Z.G. acknowledges the support of the Research Council of Norway via the grant 213474/F20, and the National Science Foundation via the grant DMS 1212023.

\section{REFERENCES}

[1] L. Caffarelli, R. Kohn, and L. Nirenberg. Partial regularity of suitable weak solutions of the Navier-Stokes equations. Comm. Pure Appl. Math., 35(6):771, 1982.

[2] D. C. Chang, G. Dafni, and E.M. Stein. Hardy spaces, $B M O$, and boundary value problems for the Laplacian on a smooth domain in $\mathbb{R}^{n}$. Trans. Amer. Math. Soc., 351(4):1605-1661, 1999.

[3] C. Chen, R. M. Strain, T. Tsai, and H.T. Yau. Lower bounds on the blow-up rate of the axisymmetric Navier-Stokes equations. II. Comm. Partial Differential Equations, 34(1-3):203-232, 2009.

[4] C. Chen, R. M. Strain, H.T. Yau, and T. Tsai. Lower bound on the blow-up rate of the axisymmetric Navier-Stokes equations. Int. Math. Res. Not. IMRN, (9):16-31, 2008.

[5] R. Coifman, P.L. Lions, Y. Meyer, and S. Semmes. Compensated compactness and Hardy spaces. J. Math. Pures Appl. (9), 72(3):247286, 1993.

[6] P. Constantin. Navier-Stokes equations and area of interfaces. Comm. Math. Phys., 129(2):241-266, 1990.

[7] P. Constantin and C. Foias. Navier-Stokes equations. Chicago Lectures in Mathematics. University of Chicago Press, Chicago, IL, 1988.

[8] R. Dascaliuc and Z. Grujić. Vortex stretching and criticality for the three-dimensional Navier-Stokes equations. J. Math. Phys., 53, 2012.

[9] M. Sodin F. Nazarov and A. Vol'berg. The geometric Kannan-Lovaśz-Simonovits lemma, dimension-free estimates for the distribution function of the values of polynomials, and the distribution of the zeros of random analytic functions. St. Petersburg Math. J., 14(2):351-366, 2003.

[10] C. Fefferman. Characterizations of bounded mean oscillation. Bull. Amer. Math. Soc., 77:587-588, 1971.

[11] C. Fefferman and E. M. Stein. $H^{p}$ spaces of several variables. Acta Math., 129(3-4):137-193, 1972.

[12] Z. Grujić. The geometric structure of the super-level sets and regularity for 3D Navier-Stokes equations. Indiana Univ. Math. J., 50:1309-1317, 2001.

[13] Z. Grujić. A geometric measure-type regularity criterion for solutions to the 3D Navier-Stokes equations. Nonlinearity, 26:289-296, 2013.

[14] Z. Grujić and R. Guberović. A regularity criterion for the 3D NSE in a local version of the space of functions of bounded mean oscillations. Ann. Inst. H. Poincaré Anal. Non Linéaire, 27(2):773-778, 2010.

[15] T.. Hou and R. Li. Nonexistence of locally self-similar blow-up for the 3D incompressible Navier-Stokes equations. Discrete Contin. Dyn. Syst., 18(4):637-642, 2007.

[16] T. Iwaniec and A. Verde. On the operator $L(f)=f l o g|f|$. J. Funct. Anal., 169(2):391-420, 1999. 
[17] G. Koch, N. Nadirashvili, G. A. Seregin, and V. Šverák. Liouville theorems for the Navier-Stokes equations and applications. Acta Math., 203(1):83-105, 2009.

[18] P. L. Lions. Mathematical topics in fluid mechanics. Vol. 1, volume 3 of Oxford Lecture Series in Mathematics and its Applications. The Clarendon Press Oxford University Press, New York, 1996. Incompressible models, Oxford Science Publications.

[19] J. Nečas, M. Ružička, and V. Šverák. On Leray's self-similar solutions of the Navier-Stokes equations. Acta Math., 176(2):283-294, 1996.

[20] G. Seregin and V. Šverák. On type I singularities of the local axi-symmetric solutions of the Navier-Stokes equations. Comm. Partial Differential Equations, 34(1-3):171-201, 2009.

[21] E. M. Stein, editor. Beijing lectures in harmonic analysis, volume 112 of Annals of Mathematics Studies, Princeton, NJ, 1986. Princeton University Press.

[22] E. M. Stein. Harmonic analysis: real-variable methods, orthogonality, and oscillatory integrals, volume 43 of Princeton Mathematical Series. Princeton University Press, Princeton, NJ, 1993.

[23] T. Tsai. Erratum: "On Leray's self-similar solutions of the Navier-Stokes equations satisfying local energy estimates" [Arch. Rational Mech. Anal. 143 (1988), no. 1, 29-51; MR1643650 (99j:35171)]. Arch. Ration. Mech. Anal., 147(4):363, 1999. 